\title{
HEIGHT BOUND AND PREPERIODIC POINTS FOR JOINTLY REGULAR FAMILIES OF RATIONAL MAPS
}

\author{
Chong Gyu Lee
}

\begin{abstract}
Silverman [14] proved a height inequality for a jointly regular family of rational maps and the author [10] improved it for a jointly regular pair. In this paper, we provide the same improvement for a jointly regular family: let $h: \mathbb{P}_{\mathbb{Q}}^{n} \rightarrow \mathbb{R}$ be the logarithmic absolute height on the projective space, let $r(f)$ be the $D$-ratio of a rational map $f$ which is defined in [10] and let $\left\{f_{1}, \ldots, f_{k} \mid f_{l}: \mathbb{A}^{n} \rightarrow \mathbb{A}^{n}\right\}$ be a finite set of polynomial maps which is defined over a number field $K$. If the intersection of the indeterminacy loci of $f_{1}, \ldots, f_{k}$ is empty, then there is a constant $C$ such that

$$
\sum_{l=1}^{k} \frac{1}{\operatorname{deg} f_{l}} h\left(f_{l}(P)\right)>\left(1+\frac{1}{r}\right) f(P)-C \quad \text { for all } P \in \mathbb{A}^{n}
$$

where $r=\max _{l=1, \ldots, k}\left(r\left(f_{l}\right)\right)$.
\end{abstract}

\section{Introduction}

Let $K$ be a number field and let $h: \mathbb{P}_{\bar{K}}^{n} \rightarrow \mathbb{R}$ be the logarithmic absolute height function on the projective space. If $f: \mathbb{P}_{K}^{n} \rightarrow \mathbb{P}_{K}^{n}$ is a morphism defined over a number field $K$, then we can make a good estimate of $h(P)$ with $h(f(P))$. Define the degree of $f$ to be the number induced by the linear operator $f^{*}$ on $\operatorname{Pic}\left(\mathbb{P}^{n}\right)=\mathbb{Z}$ :

$$
f^{*} H=\operatorname{deg} f \cdot H \text { on } \operatorname{Pic}\left(\mathbb{P}^{n}\right) .
$$

Then, the functorial property of the Weil height machine will prove the Northcott's theorem. The author refers [16, Theorem B.3.2] to the reader for the details of the Weil height machine.

Theorem 1.1 (Northcott [12]). If $f: \mathbb{P}_{K}^{n} \rightarrow \mathbb{P}_{K}^{n}$ is a morphism defined over a number field $K$, then there are two constants $C_{1}$ and $C_{2}$, which are independent of point $P$, such that

$$
\frac{1}{\operatorname{deg} f} h(f(P))+C_{1}>h(P)>\frac{1}{\operatorname{deg} f} h(f(P))-C_{2}
$$

Received April 27, 2010; Revised August 9, 2011.

2010 Mathematics Subject Classification. Primary 37P30; Secondary 11G50, 32H50, 37P05.

Key words and phrases. height, rational map, preperiodic points, jointly regular family. 
for all $P \in \mathbb{P} \frac{n}{K}$.

If $f$ is not a morphism but a rational map, then the functoriality of the Weil height machine breaks down: the two height functions $h_{f^{*} H}(P)$ and $h_{H}(f(P))$ are not equivalent. Hence, Northcott's Theorem is not valid for rational maps (However, we still have $h(P)>\frac{1}{\operatorname{deg} f} h(f(P))-C_{2}$ by the triangular inequality. See [16, Proposition B.7.1]).

For example, consider a polynomial map, one of popular objects in complex dynamics. Define

$$
f:=\left(f_{1}, \ldots, f_{n}\right): \mathbb{A}_{K}^{n} \rightarrow \mathbb{A}_{K}^{n},
$$

where $f_{1}, \ldots, f_{n}$ are homogeneous polynomials of degree $d$. We may consider $f$ as a rational map on $\mathbb{P}_{K}^{n}$ : let $P \in \mathbb{P}_{K}^{n}$. We define $f(P)$ to be the following limit value if it exists.

$$
f(P):=\lim _{\substack{Q \rightarrow P \\ Q \in \mathbb{A}_{K}^{n}}} f(Q) .
$$

We call it the meromorphic extension of $f$. In general, the meromorphic extension of $f$ is not a morphism. So, we need other way to find an upper bound of $h(P)$.

Silverman suggested a way of constructing an upper bound of $h(P)$ when we have a special family of polynomial maps.

Definition 1.2. Let $S=\left\{f_{1}, \ldots, f_{k} \mid f_{l}: \mathbb{P} \frac{n}{K} \rightarrow \mathbb{P} \frac{n}{K}\right\}$ be a finite set of rational maps defined over a number field $K$ and let $I(f)$ be the indeterminacy locus of $f$. We say that $S$ is jointly regular when

$$
\bigcap_{l=1}^{k} I\left(f_{l}\right)=\emptyset .
$$

We also say that a finite set of polynomial maps $S^{\prime}=\left\{g_{1}, \ldots, g_{k} \mid g_{l}: \mathbb{A}_{K}^{n} \rightarrow\right.$ $\left.\mathbb{A}_{K}^{n}\right\}$ is jointly regular if the set of rational maps

$$
S=\left\{f_{l}: \mathbb{P}^{n} \rightarrow \mathbb{P}^{n} \mid f_{l} \text { is the meromorphic extension of } g_{l} \in S^{\prime}\right\}
$$

is jointly regular.

Theorem 1.3 ([14], Theorem 3). Let $\left\{f_{1}, \ldots, f_{k} \mid f_{l}: \mathbb{A}_{K}^{n} \rightarrow \mathbb{A}_{K}^{n}\right\}$ be a jointly regular family of polynomial maps defined over a number field $K$. Then, there is a constant $C$ satisfying

for all $P \in \mathbb{A} \frac{n}{K}$.

$$
\sum_{l=1}^{k} \frac{1}{\operatorname{deg} f_{l}} h\left(f_{l}(P)\right)>h(P)-C
$$

In this paper, we will improve Theorem 1.3 using the $D$-ratio. The $D$-ratio requires new concepts to be defined so that we will state the main theorem without the definition of the $D$-ratio first and will introduce the $D$-ratio in Definition 2.12 later. 
Theorem 1.4. Let $H$ be a hyperplane of $\mathbb{P}^{n}$, let $\mathbb{A}^{n}=\mathbb{P}^{n} \backslash H$, let $S=$ $\left\{f_{1}, \ldots, f_{k} \mid f_{l}: \mathbb{A}_{K}^{n} \rightarrow \mathbb{A}_{K}^{n}\right\}$ be a jointly regular family of polynomial maps defined over a number field $K$, let $r(f)$ be the D-ratio of $f$ and let $r=$ $\max _{l=1, \ldots, k}\left(r\left(f_{l}\right)\right)$. Suppose that $S$ has at least two elements. Then, there is a constant $C$ satisfying

$$
\sum_{l=1}^{k} \frac{1}{\operatorname{deg} f_{l}} h\left(f_{l}(P)\right)>\left(1+\frac{1}{r}\right) h(P)-C
$$

for all $P \in \mathbb{A} \frac{n}{K}$.

This theorem improves Silverman's result for preperiodic points [14, Theorem 4], which is exactly same with Theorem 1.5 except the description of $\delta_{S}$.

Theorem 1.5. Let $S=\left\{f_{1}, \ldots, f_{k} \mid f_{l}: \mathbb{A}_{K}^{n} \rightarrow \mathbb{A}_{K}^{n}\right\}$ be a jointly regular family of polynomial maps, let $f\left(f_{l}\right)$ be the D-ratio of $f_{l}$ and let $\Phi$ be the monoid of polynomial maps generated by $S$. Define

$$
\delta_{S}:=\left(\frac{1}{1+1 / r}\right) \sum_{l=1}^{k} \frac{1}{\operatorname{deg} f_{l}},
$$

where $r=\max _{l=1, \ldots, k}\left(r\left(f_{l}\right)\right)$.

If $\delta_{S}<1$, then

$$
\operatorname{Preper}(\Phi):=\bigcap_{f \in \Phi} \operatorname{Preper}(f) \subset \mathbb{A} \frac{n}{K}
$$

is a set of bounded height.

From now on, we will let $K$ be a number field, let $H$ be a hyperplane on $\mathbb{P}^{n}$ and let $\mathbb{A}^{n}=\mathbb{P}^{n} \backslash H$ be an affine space. We also let $f: \mathbb{A}^{n} \rightarrow \mathbb{A}^{n}$ be a polynomial map and let $I(f)$ be the indeterminacy locus of $f$ unless stated otherwise.

Acknowledgements. It is a part of my Ph. D. dissertation. I would like to thank my advisor Joseph H. Silverman for his overall advice. Also, thanks to the referee for his/her priceless help.

\section{Preliminaries}

We need two main ingredients, the resolution of indeterminacy and the $D$ ratio of polynomial maps. For details, the author refers [1] and [3, II.7] for blowups and the resolution of indeterminacy, and [10] for the $D$-ratio.

\subsection{Blowup and the resolution of indeterminacy}

We have the general theorem of the resolution of indeterminacy, which is a corollary of the theorem of the resolution of singularity. 
Theorem 2.1 (Resolution of indeterminacy). Let $f: V \rightarrow W$ be a rational map between proper varieties such that $V$ is nonsingular. Then there is a proper nonsingular variety $\widetilde{V}$ with a birational morphism $\pi: \widetilde{V} \rightarrow V$ such that $\phi=f \circ \pi: \widetilde{V} \rightarrow W$ is a morphism:

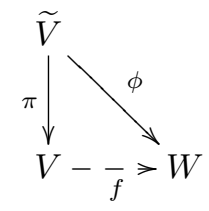

For notational convenience, we will define the following.

Definition 2.2. Let $f: \mathbb{P}^{n} \rightarrow \mathbb{P}^{n}$ be a rational map and let $V$ be a blowup of $\mathbb{P}^{n}$ with a birational morphism $\pi: V \rightarrow \mathbb{P}^{n}$. We say that a pair $(V, \pi)$ is $a$ resolution of indeterminacy of $f$ if

$$
f \circ \pi: V \rightarrow \mathbb{P}^{n}
$$

is extended to a morphism. And we call the extended morphism $\phi:=f \circ \pi$ a resolved morphism of $f$.

Using Hironaka's Theorem (Theorem 2.5), we will observe the relation between the resolution of indeterminacy and the indeterminacy locus of $f$.

Definition 2.3. Let $\pi: V \rightarrow \mathbb{P}^{n}$ be a birational morphism. Then, we say that a closed subscheme $\mathfrak{I}$ of $\mathbb{P}^{n}$ is the center scheme of $\pi$ if the ideal sheaf $\mathcal{S}$ corresponding to $\mathfrak{I}$ generates $V$ :

$$
V=\operatorname{Proj}\left(\bigoplus_{d \geq 0} \mathcal{S}^{d}\right)
$$

Definition 2.4. Let $\pi: W \rightarrow V$ be a birational morphism. We say that $\pi$ is a monoidal transformation if its center scheme is a smooth irreducible subvariety of $V$. We say that $W$ is a successive blowup of $V$ if the corresponding birational map $\pi: W \rightarrow V$ is a composition of monoidal transformations.

Theorem 2.5 (Hironaka). Let $f: X \rightarrow Y$ be a rational map between proper varieties such that $V$ is nonsingular. Then, there is a finite sequence of proper varieties $X_{0}, \ldots, X_{r}$ such that

(1) $X_{0}=X$,

(2) $\rho_{i}: X_{i} \rightarrow X_{i-1}$ is a monoidal transformation,

(3) If $T_{i}$ is the center scheme of $\pi_{i}$, then $\rho_{0} \circ \cdots \circ \rho_{i}\left(T_{i}\right) \subset I(f)$ on $X$,

(4) $f$ is extended to a morphism $\tilde{f}: X_{r} \rightarrow Y$ on $X_{r}$,

(5) Consider the composition of all monoidal transformation $\rho: X_{m} \rightarrow$ $X$. Then, the underlying subvariety of the center scheme $T$ of $\rho, a$ subvariety made by the zero set of the ideal sheaf corresponding to $T$, is exactly $I(f)$. 
Proof. See [4, Question (E) and Main Theorem II].

In $\S 2.2$, we will find a basis of $\operatorname{Pic}(V)$ when $(V, \pi)$ is a resolution of indeterminacy. Especially, we need a basis consisting of irreducible divisors. However, pullbacks of divisors may not be irreducible because of the exceptional part. So, we define the proper transformation, which is usually irreducible.

Definition 2.6. Let $\pi: \widetilde{V} \rightarrow V$ be a birational morphism with center scheme $\Im$ and let $D$ be an irreducible divisor on $V$. We define the proper transformation of $D$ by $\pi$ to be

$$
\pi^{\#} D=\overline{\pi^{-1}(D \cap U)}
$$

where $U=V \backslash Z(\mathfrak{I})$ and $Z(\mathfrak{I})$ is the underlying subvariety made by the zero set of the ideal corresponding to $\mathfrak{I}$.

\subsection{The $\mathbb{A}^{n}$-effectiveness and the $D$-ratio}

The main question of this paper is to find an upper bound of $h(P)$ using $h\left(f_{l}(P)\right)$ for jointly regular family $\left\{f_{1}, \ldots, f_{k} \mid f_{l}: \mathbb{A}^{n} \rightarrow \mathbb{A}^{n}\right\}$. So, we will consider $\mathbb{A}^{n}$ as a dense open subset of $\mathbb{P}^{n}$ and fix the hyperplane $H=\mathbb{P}^{n} \backslash \mathbb{A}^{n}$ to find a basis of the Picard group of a blowup of $\mathbb{P}^{n}$ and use a special kind of divisors on a blowup of $\mathbb{P}^{n}$ to measure the height values of $P \in \mathbb{A}^{n}$. First of all, we need to clarify how to get such basis of $\operatorname{Pic}(V)$.

Proposition 2.7. Let $V$ be a successive blowup of $\mathbb{P}^{n}$ with a birational morphism $\pi: V \rightarrow \mathbb{P}^{n}$ : there are monoidal transformations $\pi_{i}: V_{i} \rightarrow V_{i-1}$ such that $V_{r}=V$ and $V_{0}=\mathbb{P}^{n}$. Let $H$ be a hyperplane on $\mathbb{P}^{n}$, let $F_{i}$ be the exceptional divisor of the blowup $\pi_{i}: V_{i} \rightarrow V_{i-1}$, let $\rho_{i}=\pi_{i+1} \circ \cdots \circ \pi_{r}$ and let $E_{i}=\rho_{i}^{\#} F_{i}$. Then, $\operatorname{Pic}(V)$ is a free $\mathbb{Z}$-module with a basis

$$
\left\{H_{V}=\pi^{\#} H, E_{1}, \ldots, E_{r}\right\} .
$$

Proof. [3, Exer.II.7.9] shows that

$$
\operatorname{Pic}(\widetilde{X}) \simeq \operatorname{Pic}(X) \oplus \mathbb{Z}
$$

if $\pi: \widetilde{X} \rightarrow X$ is a monoidal transformation. More precisely,

$$
\operatorname{Pic}(\widetilde{X})=\left\{\pi^{\#} D+n E \mid D \in \operatorname{Pic}(X)\right\}
$$

where $E$ is the exceptional divisor of $\pi$ on $\widetilde{X}$. Suppose that $X=V_{i-1}$ and $\widetilde{X}=V_{i}$ and get the desired result.

Now, we define the special kind of divisors, the $\mathbb{A}^{n}$-effective divisors.

Definition 2.8. Let $V$ be a successive blowup of $\mathbb{P}^{n}$ with a birational morphism $\pi: V \rightarrow \mathbb{P}^{n}$, let $H$ be a fixed hyperplane of $\mathbb{P}^{n}$ and let

$$
\operatorname{Pic}_{\mathbb{Q}}(V)=\mathbb{Q} H_{V} \oplus \mathbb{Q} E_{1} \oplus \cdots \oplus \mathbb{Q} E_{r}
$$

with the basis described in Proposition 2.7. We define the $\mathbb{A}^{n}$-effective cone to be

$$
\operatorname{AFE}(V):=\mathbb{Q}^{\geq 0} H_{V} \oplus \mathbb{Q}^{\geq 0} E_{1} \oplus \cdots \oplus \mathbb{Q}^{\geq 0} E_{r},
$$


where $\mathbb{Q}^{\geq 0}$ is the set of nonnegative rational numbers. We say a divisor $D$ of $V$ is $\mathbb{A}^{n}$-effective if the linear equivalence class of $D$ is contained in $\operatorname{AFE}(V)$ and denote it by

$$
D \succ 0 \text {. }
$$

Moreover, on $\operatorname{Pic}_{\mathbb{Q}}(V)$, we write

$$
D_{1} \succ D_{2}
$$

if $D_{1}-D_{2}$ is $\mathbb{A}^{n}$-effective.

The next proposition will explain why we define the " $\mathbb{A}^{n}$-effectiveness". Namely, the height functions corresponding to $\mathbb{A}^{n}$-effective divisors will have nice properties on $\mathbb{A}^{n}$.

Proposition 2.9. Let $V$ be a successive blowup of $\mathbb{P}^{n}$ with a birational morphism $\pi: V \rightarrow \mathbb{P}^{n}$ and let $D, D_{i}$ be divisors on $V$.

(1) (Effectiveness) If $D$ is $\mathbb{A}^{n}$-effective, then $D$ is effective.

(2) (Boundedness) If $D$ is $\mathbb{A}^{n}$-effective, then $h_{D}(P)$ is bounded below on $V \backslash\left(H_{V} \cup\left(\bigcup_{i=1}^{r} E_{i}\right)\right)$.

(3) (Transitivity) If $D_{1} \succ D_{2}$ and $D_{2} \succ D_{3}$, then $D_{1} \succ D_{3}$.

(4) (Funtoriality) If $\rho: W \rightarrow V$ is a monoidal transformation and $D_{1} \succ D_{2}$, then $\rho^{*} D_{1} \succ \rho^{*} D_{2}$.

Proof. See [10, Proposition 3.3].

In Section 1, we introduce the main theorem without the definition of the $D$-ratio because it requires the $\mathbb{A}^{n}$-effectiveness. Now, we are ready to define the $D$-ratio, one of main ingredients of this paper.

Definition 2.10. Let $f: \mathbb{P}^{n} \rightarrow \mathbb{P}^{n}$ be a rational map such that $I(f) \subset H$, let $\left(V, \pi_{V}\right)$ be a resolution of indeterminacy of $f$ and let $\phi_{V}$ be a resolved morphism so that the following diagram commutes:

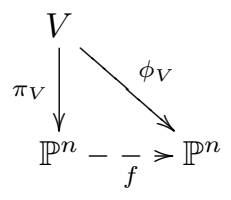

Suppose that

$$
\pi_{V}^{*} H=a_{0} H_{V}+\sum_{i=1}^{r} a_{i} E_{i} \text { and } \phi_{V}^{*} H=b_{0} H_{V}+\sum_{i=1}^{r} b_{i} E_{i},
$$

where $a_{i}, b_{i}$ are nonnegative integers. If $b_{i} \neq 0$ for all $i$ satisfying $a_{i} \neq 0$, we define the $D$-ratio of $\phi_{V}$ to be

$$
r\left(\phi_{V}\right)=\operatorname{deg} \phi_{V} \cdot \max _{a_{i} \neq 0}\left(\frac{a_{i}}{b_{i}}\right) .
$$


If there is an index $i$ satisfying $a_{i} \neq 0$ and $b_{i}=0$, define

$$
r\left(\phi_{V}\right)=\infty \text {. }
$$

The readers might concern if the $D$-ratio is only defined for resolved morphisms. The following lemma will allow us to define the $D$-ratio for the rational maps.

Lemma 2.11. Let $\left(V, \pi_{V}\right)$ and $\left(W, \pi_{V}\right)$ be resolutions of indeterminacy of $f$ with resolved morphisms $\phi_{V}=f \circ \pi_{V}$ and $\phi_{W}=f \circ \pi_{W}$ respectively:

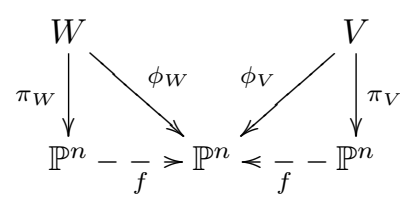

Then, we have

$$
r\left(\phi_{V}\right)=r\left(\phi_{W}\right)
$$

Proof. See [10, Lemma 4.3].

Definition 2.12. Let $f: \mathbb{P}^{n} \rightarrow \mathbb{P}^{n}$ be a rational map with $I(f) \subset H$. Then, we define the $D$-ratio of $f$ to be

$$
r(f)=r\left(\phi_{V}\right)
$$

for any resolution of indeterminacy $\left(V, \pi_{V}\right)$ of $f$ with resolved morphism $\phi_{V}$.

Proposition 2.13. Let $f, g: \mathbb{P}^{n} \rightarrow \mathbb{P}^{n}$ be rational maps such that $I(f), I(g) \subset$ H. Then,

(1) $r(f)=1$ if and only if $f$ is a morphism.

(2) $r(f) \in[1, \infty]$.

(3) $\frac{r(f)}{\operatorname{deg} f} \cdot \frac{r(g)}{\operatorname{deg} g} \geq \frac{r(g \circ f)}{\operatorname{deg}(g \circ f)}$.

Proof. See [10, Proposition 4.5, Theorem 5.2].

Example 2.14. Let $f: \mathbb{A}^{n} \rightarrow \mathbb{A}^{n}$ be a polynomial automorphism with the inverse map $f^{-1}: \mathbb{A}^{n} \rightarrow \mathbb{A}^{n}$. Then, $r(f)=\operatorname{deg} f \times \operatorname{deg} f^{-1}$ (For details, see [9]). For example, a Hénon map

$$
f_{H}(x, y, z)=\left(z, x+z^{2}, y+x^{2}\right)
$$

is a regular polynomial automorphism with the inverse map

$$
f_{H}^{-1}(x, y, z)=\left(y-x^{2}, z-\left(y-x^{2}\right)^{2}, x\right) .
$$

Thus,

$$
r\left(f_{H}\right)=r\left(f_{H}^{-1}\right)=\operatorname{deg} f_{H} \times \operatorname{deg} f_{H}^{-1}=2 \times 4=8 .
$$


Example 2.15. Let $f[x, y, z]=\left[x^{2}, y z, z^{2}\right]$. Then, the indeterminacy locus of $f$ consists of one point $P=[0,1,0]$. Then, the blowup $V$ along closed scheme corresponding ideal sheaf $\left(z, x^{2}\right)$ will resolve indeterminacy, which is a successive blowup along $P$ and $H^{\#} \cap F_{1}$, where $F_{1}$ is the exceptional divisor of the first blowup.

Let $E_{1}$ be the proper transformation of $F_{1}$ and let $E_{2}$ be the exceptional divisor of the second blowup:
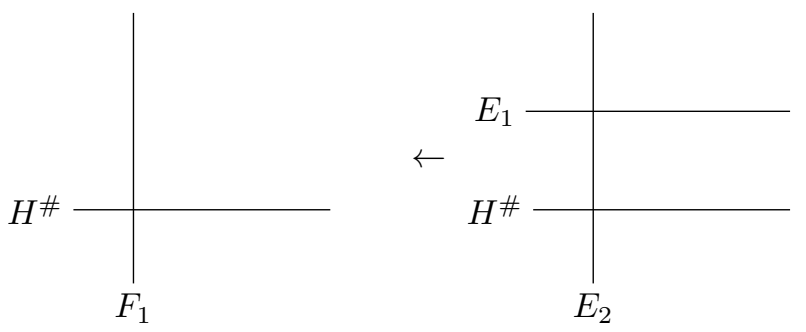

Then, the following intersection numbers are easily calculated:

$E_{2}^{2}=-1, E_{1}^{2}=-2,\left(H^{\#}\right)^{2}=-1, H^{\#} \cdot E_{1}=0$ and $H^{\#} \cdot E_{2}=E_{1} \cdot E_{2}=1$.

Furthermore, by the projection formula and the exact calculation of $\phi_{*}$, we get

$$
\begin{gathered}
H^{\#} \cdot \phi^{*} H=\phi_{*} H^{\#} \cdot H=0, \\
E_{1} \cdot \phi^{*} H=\phi_{*} E_{1} \cdot H=0, \\
E_{2} \cdot \phi^{*} H=\phi_{*} E_{2} \cdot H=1 .
\end{gathered}
$$

Since $\operatorname{Pic}(V)=\left\langle H^{\#}, E_{1}, E_{2}\right\rangle$, we may assume that

$$
\phi^{*} H=a H^{\#}+b E_{1}+c E_{2}
$$

for some integers $a, b$ and $c$. Then, by previous facts,

$$
\phi^{*} H \cdot H^{\#}=-a+c=0, \quad \phi^{*} H \cdot E_{1}=a-2 b=0 .
$$

Therefore,

$$
\phi^{*} H=2 H^{\#}+E_{1}+2 E_{2}, \quad \pi^{*} H=H^{\#}+E_{1}+2 E_{2}
$$

and hence

$$
r(f)=2 \times 1=2 .
$$

\section{Jointly regular families of rational maps}

Proof of Theorem 1.4. For notational convenience, let

- $d_{l}=\operatorname{deg} f_{l}$.

- $r_{l}=r\left(f_{l}\right)$.

- $\left(V_{l}, \pi_{l}\right)$ be a resolution of indeterminacy of $f_{l}$ constructed by Theorem 2.5: assume that $\pi_{l}$ is a composition of monoidal transformations and $\left\{\pi_{l}^{\#} H=H_{V_{l}}, E_{l 1}, \ldots, E_{l s_{l}}\right\}$ is the basis of $\operatorname{Pic}\left(V_{l}\right)$ given by Proposition 2.7 . 
- $\phi_{l}$ be the resolved morphism of $f_{l}$ on $V_{l}$.

$$
\begin{aligned}
& \pi_{l}^{*} H=a_{0} H_{V_{l}}+\sum_{i=1}^{s_{l}} a_{l i} E_{l i} \text { and } \phi_{l}^{*} H=b_{0} H_{V_{l}}+\sum_{i=1}^{s_{l}} b_{l i} E_{l i} \\
& \text { in } \operatorname{Pic}\left(V_{l}\right)=\mathbb{Z} \pi_{l}^{\#} H \oplus \mathbb{Z} E_{l 1} \oplus \cdots \oplus \mathbb{Z} E_{l s_{l}} .
\end{aligned}
$$

We can easily check that $a_{0}=1$ and $b_{0}=d_{l}$ from $\pi_{l *} \pi_{l}^{*} H=H$ and $\pi_{l *} \phi_{l}^{*} H=\operatorname{deg} \phi_{l} \cdot H$. For details, see [10, Proposition 4.5(2)].

Let $T_{l}$ be the center scheme of blowup for $V_{l}$ and let $W$ be the blowup of $\mathbb{P}^{n}$ whose center scheme is $\sum T_{l}$. Then, $W$ is a blowup of $V_{l}$ for all $l$. Furthermore, since the underlying set of $T_{l}$ is exactly $I\left(f_{l}\right)$, the underlying set of $\sum T_{l}=\cup I\left(f_{l}\right)$. Let $\rho_{l}: W \rightarrow V_{l}, \pi_{W}$ be a composition of monoidal transformations:

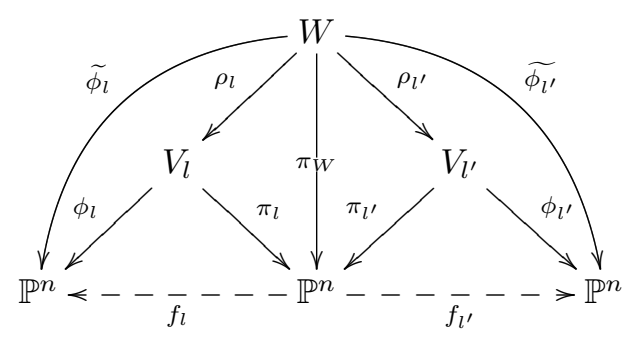

Then, still $W$ is a blowup of $\mathbb{P}^{n}$ and hence $\operatorname{Pic}(W)$ is generated by $\pi_{W}^{\#} H$ and the irreducible components $F_{j}$ of the exceptional divisor:

$$
\operatorname{Pic}(W)=\mathbb{Z} \pi_{W}^{\#} H \oplus \mathbb{Z} F_{1} \oplus \cdots \oplus \mathbb{Z} F_{s} .
$$

Thus, we can represent $\pi_{W}^{*} H$ as follows.

$$
\pi_{W}^{*} H=\pi_{W}^{\#} H+\sum_{j=1}^{s} \alpha_{j} F_{j}
$$

To describe $\phi_{l}^{*} H$ precisely, define

$\mathcal{I}_{l}=\left\{1 \leq j \leq s \mid \pi_{W}\left(F_{j}\right) \subset I\left(f_{l}\right)\right\} \quad$ and $\quad \mathcal{I}_{l}^{c}=\left\{1 \leq j \leq s \mid \pi_{W}\left(F_{j}\right) \not \subset I\left(f_{l}\right)\right\}$.

By definition, it is clear that

$$
\mathcal{I}_{l} \cup \mathcal{I}_{l}^{c}=\{1, \ldots, s\} \quad \text { and } \quad \mathcal{I}_{l} \cap \mathcal{I}_{l}^{c}=\emptyset .
$$

Thus, we can say

$$
\widetilde{\phi}_{l}^{*} H=d_{l} \pi_{W}^{\#} H+\sum_{j=1}^{s} \beta_{l j} F_{j}=d_{l} \pi_{W}^{\#} H+\sum_{j \in \mathcal{I}_{l}^{c}} \beta_{l j} F_{j}+\sum_{j \in \mathcal{I}_{l}} \beta_{l j} F_{j} .
$$

We have the following lemmas which clarify the relation between coefficients of $F_{j}$ 's. 
Lemma 3.1.

$$
\bigcup_{l=1}^{k} \mathcal{I}_{l}=\bigcup_{l=1}^{k} \mathcal{I}_{l}^{c}=\{1, \ldots, s\}
$$

Proof. $\bigcup_{l} \mathcal{I}_{l}=\{1, \ldots, s\}$ is clear; because the underlying set of the center scheme of $W$ is $\cup I\left(f_{l}\right), \cup \pi_{W}\left(F_{j}\right)=\pi_{W}\left(\cup F_{j}\right)=\cup I\left(f_{l}\right)$.

Suppose $\bigcup_{l} \mathcal{I}_{l}^{c} \subsetneq\{1, \ldots, s\}$. Then, there is an index $l_{0}$ satisfying $\pi_{W}\left(F_{l_{0}}\right) \subset$ $I\left(f_{l}\right)$ for all $l$. This implies $\pi_{W}\left(F_{l_{0}}\right) \subset I\left(f_{l}\right)$ for all $l$ and hence $\emptyset \neq \pi_{W}\left(F_{l_{0}}\right) \subset$ $\bigcap_{l} I\left(f_{l}\right)$ which contradicts to the assumption that $S$ is jointly regular.

Lemma 3.2. Let $\alpha_{j}$ and $\beta_{l j}$ be the coefficients of $F_{j}$ in $\pi_{V}^{*} H$ and $\widetilde{\phi}_{l}^{*} H$ respectively. Then,

Especially, if $j \in \mathcal{I}_{l}^{c}$, then

$$
d_{l} \frac{\alpha_{j}}{\beta_{l j}} \leq r_{l}
$$

$$
d_{l} \alpha_{j}=\beta_{l j}
$$

Proof. By definition of the $D$-ratio, the first inequality is clear:

$$
r_{l}=d_{l} \cdot \max _{i}\left(\frac{\alpha_{i}}{\beta_{l i}}\right) \geq d_{l} \cdot \frac{\alpha_{j}}{\beta_{l j}} .
$$

Now, suppose that

$$
\begin{aligned}
\rho_{l}^{*} \pi_{l}^{\#} H & =\kappa_{l 0} \pi_{W}^{\#} H+\sum_{j=1}^{s} \kappa_{l j} F_{j}=\kappa_{l j} \pi_{W}^{\#} H+\sum_{j \in \mathcal{I}_{l}^{c}} \kappa_{l j} F_{j}+\sum_{j \in \mathcal{I}_{l}} \kappa_{l j} F_{j}, \\
\rho_{l}^{*} E_{l i} & =\lambda_{l i 0} \pi_{W}^{\#} H+\sum_{j=1}^{s} \lambda_{l i j} F_{j}=\lambda_{l i 0} \pi_{W}^{\#} H+\sum_{j \in \mathcal{I}_{l}^{c}} \lambda_{l i j} F_{j}+\sum_{j \in \mathcal{I}_{l}} \lambda_{l i j} F_{j} .
\end{aligned}
$$

We can easily calculate some of $\kappa_{l j}, \lambda_{l i j}$ :

(a) $\lambda_{l i 0}=0$ for all $i=1, \ldots, s_{l}$.

Because $\pi_{W}\left(\rho_{l}^{*} E_{i}\right) \subset \cup I\left(f_{l}\right)$, we get $\left(\pi_{W}\right)_{*}\left(\rho_{l}^{*} E_{i}\right)=0$. On the other hand, $\pi_{W} *$ eliminates all $F_{j}$ so that

$$
\pi_{W *}\left(\lambda_{l i 0} \pi_{W}^{\#} H+\sum_{j=1}^{s} \lambda_{l i j} F_{j}\right)=\lambda_{l i 0} H .
$$

Hence, $\lambda_{l i 0}=0$.

(b) $\kappa_{l 0}=1$.

We have

$$
\pi_{W *}\left(\pi_{W}^{*} H\right)=H
$$

because $\pi_{W}$ is one-to-one outside of the center of blowup of $W$. Therefore,

$$
\pi_{W *}\left(\rho_{l}^{*} \pi_{l}^{\#} H\right)=\pi_{W *}\left(\pi_{W}^{*} H-\sum_{j=1}^{s} a_{l i} \rho_{l}^{*} E_{l i}\right)=H .
$$


On the other hand, $\pi_{*}$ eliminates all $F_{j}$ so that

$$
\pi_{W *}\left(\kappa_{l 0} \pi_{W}^{\#} H+\sum_{j=1}^{s} \kappa_{l j} F_{j}\right)=\kappa_{l 0} H .
$$

Hence, $\kappa_{l 0}=1$.

(c) $\lambda_{l i j}=0$ for all $j \in \mathcal{I}_{l}^{c}$.

Because $\pi_{l}\left(E_{l i}\right) \subset I\left(f_{l}\right)$ and $\pi_{W}\left(F_{j}\right) \not \subset I\left(f_{l}\right)$ for any $j \in \mathcal{I}_{l}^{c}$, the multiplicity of $\rho_{l}\left(F_{j}\right)$ on $E_{l}$ is zero and hence $\gamma_{l i j}=0$. Thus, we can say

$$
\rho_{l}^{*} E_{l i}=\sum_{j \in \mathcal{I}_{l}} \lambda_{l i j} F_{j} .
$$

Let's complete the proof of Lemma 3.2. Since $\widetilde{\phi}_{l}=\rho_{l} \circ \phi_{l}$ and $\pi_{W}=\rho_{l} \circ \pi_{l}$, we can use (a), (b) and (c) to get the description of $\pi_{W *}$ and $\widetilde{\phi}_{l}^{*} H$ :

$$
\begin{aligned}
\pi_{W}^{*} H= & \rho_{l}^{*} \pi_{l}^{*} H \\
= & \rho_{l}^{*}\left(\pi_{l}^{\#} H+\sum_{i=1}^{s_{l}} a_{l i} E_{l i}\right) \\
= & \left(\kappa_{l 0} \pi_{W}^{\#} H+\sum_{j \in \mathcal{I}_{l}} \kappa_{l j} F_{j}+\sum_{j \in \mathcal{I}_{l}^{c}} \kappa_{l j} F_{j}\right) \\
& +\sum_{i=1}^{s_{l}} a_{l i}\left(\lambda_{l i 0} \pi_{W}^{\#} H+\sum_{j \in \mathcal{I}_{l}^{c}} \lambda_{l i j} F_{j}+\sum_{j \in \mathcal{I}_{l}} \lambda_{l i j} F_{j}\right) \\
= & \left(\pi_{W}^{\#} H+\sum_{j \in \mathcal{I}_{l}} \kappa_{l j} F_{j}+\sum_{j \in \mathcal{I}_{l}^{c}} \kappa_{l j} F_{j}\right)+\sum_{i=1}^{s_{l}} a_{l i}\left(\sum_{j \in \mathcal{I}_{l}} \lambda_{l i j} F_{j}\right) \\
= & \pi_{W}^{\#} H+\sum_{j \in \mathcal{I}_{l}^{c}} \kappa_{l j} F_{j}+\sum_{j \in \mathcal{I}_{l}}\left(\sum_{i=0}^{s_{l}} \kappa_{l j}+a_{l i} \lambda_{l i j}\right) F_{j}
\end{aligned}
$$

and

$$
\begin{aligned}
\widetilde{\phi}_{l}^{*} H= & \rho_{l}^{*} \phi_{l}^{*} H \\
= & \rho_{l}^{*}\left(d_{l} \pi_{l}^{\#} H+\sum_{i=1}^{s_{l}} b_{l i} E_{l i}\right) \\
= & d_{l}\left(\kappa_{l 0} \pi_{W}^{\#} H+\sum_{j \in \mathcal{I}_{l}} \kappa_{l j} F_{j}+\sum_{j \in \mathcal{I}_{l}^{c}} \kappa_{l j} F_{j}\right) \\
& +\sum_{i=1}^{s_{l}} b_{l i}\left(\lambda_{l i 0} \pi_{W}^{\#} H+\sum_{j \in \mathcal{I}_{l}^{c}} \lambda_{l i j} F_{j}+\sum_{j \in \mathcal{I}_{l}} \lambda_{l i j} F_{j}\right)
\end{aligned}
$$




$$
\begin{aligned}
& =d_{l}\left(\pi_{W}^{\#} H+\sum_{j \in \mathcal{I}_{l}} \kappa_{l j} F_{j}+\sum_{j \in \mathcal{I}_{l}^{c}} \kappa_{l j} F_{j}\right)+\sum_{i=1}^{s_{l}} b_{l i}\left(\sum_{j \in \mathcal{I}_{l}} \lambda_{l i j} F_{j}\right) \\
& =d_{l} \pi_{W}^{\#} H+\sum_{j \in \mathcal{I}_{l}^{c}} d_{l} \kappa_{l j} F_{j}+\sum_{j \in \mathcal{I}_{l}}\left(\sum_{i=0}^{s_{l}} d_{l} \kappa_{l j}+b_{l i} \lambda_{l i j}\right) F_{j} .
\end{aligned}
$$

Therefore,

$$
d_{l} \alpha_{j}=d_{l} \sum_{j \in \mathcal{I}_{l}^{c}} \kappa_{l j}=\beta_{j} \quad \text { for all } j \in \mathcal{I}_{l}^{c} .
$$

We now complete the proof of Theorem 1.4. Let $r=\max _{l=1, \ldots, k} r_{l}$. Note that

$$
p_{0} \pi_{W}^{\#} H+\sum_{j=1}^{s} p_{j} F_{j} \succ q_{0} \pi_{W}^{\#} H+\sum_{j=1}^{s} q_{j} F_{j}
$$

if $p_{j} \geq q_{j}$ for all $j=0, \ldots, s$. Thus,

$$
\begin{aligned}
\sum_{l=1}^{k} \frac{1}{d_{l}} \widetilde{\phi}_{l}^{*} H & =\sum_{l=1}^{k}\left[\pi_{W}^{\#} H+\sum_{j \in \mathcal{I}_{l}^{c}}\left(\frac{\beta_{l j}}{d_{l}} F_{j}\right)+\sum_{j \in \mathcal{I}_{l}}\left(\frac{\beta_{l j}}{d_{l}} F_{j}\right)\right] \\
& \succ \sum_{l=1}^{k} \pi_{W}^{\#} H+\sum_{l=1}^{k} \sum_{k \in \mathcal{I}_{l}^{c}} \alpha_{j} F_{j}+\sum_{l=1}^{k}\left(\sum_{j \in \mathcal{I}_{l}} \frac{\alpha_{j}}{r_{l}} F_{j}\right) \quad(\because \text { Lemma 3.2) } \\
& \succ k \pi_{W}^{\#} H+\sum_{l=1}^{k} \sum_{j \in \mathcal{I}_{l}^{c}} \alpha_{j} F_{j}+\sum_{l=1}^{k}\left(\sum_{j \in \mathcal{I}_{l}} \frac{\alpha_{j}}{r} F_{j}\right) \quad\left(\because r \geq r_{l}\right) \\
& \succ k \pi_{W}^{\#} H+\sum_{j=1}^{s} \alpha_{j} F_{j}+\frac{1}{r} \sum_{j=1}^{s} \alpha_{j} F_{j} \quad(\because \text { Lemma 3.1 }) \\
& \succ\left(1+\frac{1}{r}\right) \pi_{W}^{*} H \quad\left(\because k>1, r \geq r_{l} \geq 1\right)
\end{aligned}
$$

and hence

$$
D=\sum_{l=1}^{k} \frac{1}{d_{l}} \widetilde{\phi}_{l}^{*} H-\left(1+\frac{1}{r}\right) \pi_{W}^{*} H
$$

is an $\mathbb{A}^{n}$-effective divisor.

So, by Proposition $2.9, h_{D}$ is bounded below on $\pi_{W}^{-1} \mathbb{A}^{n}$. Therefore, there is a constant $C$ such that

$$
\begin{aligned}
h_{D}(Q) & =\sum_{l=1}^{k} \frac{1}{d_{l}} h_{\widetilde{\phi}_{l}^{*} H}(Q)-\left(1+\frac{1}{r}\right) h_{\pi_{W}^{*} H}(Q) \\
& =\sum_{l=1}^{k} \frac{1}{d_{l}} h_{* H}\left(\widetilde{\phi}_{l}(Q)\right)-\left(1+\frac{1}{r}\right) h_{H}\left(\pi_{W}(Q)\right)>C
\end{aligned}
$$


for all $Q \in \pi_{W}^{-1}\left(\mathbb{A}^{n}\right)(\bar{K})$. Finally, for $P=\pi_{W}(Q)$, we have $\widetilde{\phi}_{l}(Q)=f(P)$ and hence we obtain

$$
\sum_{l=1}^{k} \frac{1}{d_{l}} h_{H}(P)-\left(1+\frac{1}{r}\right) h_{H}(P)>C .
$$

Example 3.3. Let

$f_{1}=\left(z, y+z^{2}, x+\left(y+z^{2}\right)^{2}\right), \quad f_{2}=\left(x, y^{2}, z\right), \quad$ and $f_{3}=\left(x^{3}, x+y, y+z^{2}\right)$.

Their indeterminacy loci in $\mathbb{P}^{3}$ are

$$
I\left(f_{1}\right)=\{[x, y, 0,0]\}, \quad I\left(f_{2}\right)=\{[x, 0, z, 0]\}, \quad \text { and } I\left(f_{3}\right)=\{[0, y, z, 0]\} .
$$

Then, the $r\left(f_{1}\right)=8, r\left(f_{2}\right)=2$ and $r\left(f_{3}\right)=3 / 2$ (For details of the $D$-ratio calculation, see [10]). Therefore,

$$
\begin{aligned}
& h\left(\left(z, y+z^{2}, x+\left(y+z^{2}\right)^{2}\right)\right)+h\left(\left(x, y^{2}, z\right)\right)+h\left(\left(z^{3}, x+y, y+z^{2}\right)\right) \\
\geq & \left(1+\frac{1}{8}\right) h((x, y, z))-C
\end{aligned}
$$

for some constant $C$.

Corollary 3.4. Let $S$ be a jointly regular set of affine morphisms. Then,

$$
\kappa(S):=\liminf _{\substack{P \in \mathbb{A}^{n}(\overline{\mathbb{Q}}) \\ h(P) \rightarrow \infty}} \sum_{f \in S} \frac{1}{\operatorname{deg} f} \frac{h(f(P))}{h(P)} \geq 1+\frac{1}{r},
$$

where $r=\max _{f \in S} r(f)$.

Remark 3.5. Corollary 3.4 may not be the exact limit infimum values. For example, if there is a subset $S^{\prime} \subset S$ such that $S^{\prime}$ is still jointly regular and $\max _{f \in S^{\prime}} r(f)<\max _{f \in S} r(f)$, then

$$
\kappa(S) \geq \kappa\left(S^{\prime}\right) \geq 1+\frac{1}{r^{\prime}}>1+\frac{1}{r} .
$$

Example 3.6. We have the following examples for $\kappa(S)=1+\min _{f \in S}\left(\frac{1}{r(f)}\right)$.

(1) $S=\{f, g\}$ where $f, g$ are morphisms.

If $f, g$ are morphisms, then $r(f)=r(g)=1$. Therefore,

$$
\frac{1}{\operatorname{deg} f} h(f(P))+\frac{1}{\operatorname{deg} g} h(g(P))=h(P)+h(P)+O(1) .
$$

(2) $S=\left\{f, f^{-1}\right\}$ where $f$ is a regular affine automorphism and $f^{-1}$ is the inverse of $f$.

It is proved by Kawaguchi. See [6]. 


\section{An application to arithmetic dynamics}

The purpose of this section is to prove Theorem 1.5. This result is a generalization of [14, Section 4]. The proof is almost the same except one: the only difference is that we have an improved height inequality for a jointly regular family.

Fix an integer $m \geq 1$ and let $S=\left\{f_{1}, \ldots, f_{k}: \mathbb{A}_{K}^{n} \rightarrow \mathbb{A}_{K}^{n}\right\}$ be a jointly regular family defined over a number field $K$. For each $m \geq 0$, let $W_{m}$ be the collection of ordered $m$-tuples chosen from $\{1, \ldots, k\}$,

$$
W_{m}=\left\{\left(i_{1}, \ldots, i_{m}\right) \mid i_{j} \in\{1, \ldots, k\}\right\}
$$

and let

$$
W_{*}=\bigcup_{m \geq 0} W_{m} .
$$

Thus $W_{*}$ is the collection of words of $r$ symbols.

For any $I=\left(i_{1}, \ldots, i_{m}\right) \in W_{m}$, let $f_{I}$ denote the composition of corresponding polynomial maps in $S$ :

$$
f_{I}:=f_{i_{1}} \circ \cdots \circ f_{i_{m}} .
$$

Definition 4.1. We denote the monoid of rational maps generated by $S=$ $\left\{f_{1}, \ldots, f_{k}\right\}$ under composition by

$$
\Phi_{S}=\Phi:=\left\{\phi=f_{I} \mid I \in W_{*}\right\} .
$$

Let $P \in \mathbb{A}^{n}$. The $\Phi$-orbit of $P$ is defined to be

$$
\Phi(P)=\{\phi(P) \mid \phi \in \Phi\} .
$$

The set of (strongly) $\Phi$-preperiodic points is the set

$$
\operatorname{Preper}(\Phi)=\left\{P \in \mathbb{A}^{n} \mid \Phi(P) \text { is finite }\right\} \text {. }
$$

Proof of Theorem 1.5. By Theorem 1.4, we have a constant $C$ such that

$$
0 \leq\left(\frac{1}{1+\frac{1}{r}}\right) \sum_{l=1}^{k} \frac{1}{d_{l}} h\left(f_{l}(Q)\right)-h(Q)+C \quad \text { for all } Q \in \mathbb{A}^{n} .
$$

Note that if $r=\infty$, then $\left(\frac{1}{1+\frac{1}{r}}\right)=1$, then it is done because of [14, Theorem 4]. Thus, we may assume that $r$ is finite.

We define a map $\mu: W_{*} \rightarrow \mathbb{Q}$ by the following rule:

$$
\mu_{I}=\mu_{\left(i_{1}, \ldots, i_{m}\right)}=\prod d_{l}^{p_{I, l}}
$$

where $p_{I, l}=-\left|\left\{t \mid i_{t}=l\right\}\right|$. Then, by definition of $\delta_{S}$ and $\mu_{I}$, the following is true:

$$
\delta_{S}^{m}=\left[\left(\frac{r}{r+1}\right) \sum_{l=1}^{k} \frac{1}{d_{l}}\right]^{m}=\left(\frac{r}{r+1}\right)^{m} \sum_{I \in W_{m}} \frac{1}{\operatorname{deg} f_{i_{1}} \cdots \operatorname{deg} f_{i_{m}}}
$$




$$
=\left(\frac{r}{r+1}\right)^{m} \sum_{I \in W_{m}} \mu_{I}
$$

Let $P \in \mathbb{A}^{n}(\overline{\mathbb{Q}})$. Then, (1) holds for $f_{I}(P)$ for all $I \in W_{m}$ :

$$
0 \leq\left(\frac{r}{r+1}\right) \sum_{l=1}^{k} \frac{1}{d_{l}} h\left(f_{l}\left(f_{I}(P)\right)\right)-h\left(f_{I}(P)\right)+C .
$$

Hence

(2)

$$
0 \leq \sum_{m=0}^{M} \sum_{I \in W_{m}} \mu_{I}\left(\frac{r}{r+1}\right)^{m}\left[\sum_{l=1}^{k} \frac{1}{d_{l}} h\left(f_{l}\left(f_{I}(P)\right)\right)-\left(1+\frac{1}{r}\right) h\left(f_{I}(P)\right)+C\right] \text {. }
$$

The main difficulty of the inequality is to figure out the constant term. From the definition of $\delta_{S}$, we have

$$
\sum_{m=0}^{M-1}\left(\frac{r}{r+1}\right)^{m} \sum_{I \in W_{m}} \mu_{I}=\sum_{m=1}^{M} \delta_{S}^{m} \leq \frac{1}{1-\delta_{S}}
$$

Now, do the telescoping sum and most terms in (2) will be canceled:

$$
\begin{aligned}
& \left(\sum_{m=0}^{M-1} \sum_{I \in W_{m}}\left(\frac{r}{r+1}\right)^{m} \mu_{I} \sum_{l=1}^{k} \frac{1}{d_{k}} h\left(f_{l} f_{I}(P)\right)\right) \\
& -\left(\sum_{m=1}^{M} \sum_{I \in W_{m}}\left(\frac{r}{r+1}\right)^{m-1} \mu_{I} h\left(f_{I}(P)\right)\right) \\
= & \left(\sum_{m=0}^{M-1} \sum_{I \in W_{m}}\left(\frac{r}{r+1}\right)^{m} \sum_{l=1}^{k} \frac{\mu_{I}}{d_{l}} h\left(f_{l} f_{I}(P)\right)\right) \\
& -\left(\sum_{m=0}^{M-1} \sum_{I \in W_{m}} \sum_{l=1}^{k}\left(\frac{r}{r+1}\right)^{m} \frac{\mu_{I}}{d_{l}} h\left(f_{l} f_{I}(P)\right)\right) \\
= & 0 .
\end{aligned}
$$

Therefore, the remaining terms in (2) are the first term when $m=M$ and the last term when $m=0$. Thus, we get

$$
\begin{aligned}
0 & \leq\left[\sum_{I \in W_{M}}\left(\frac{r}{r+1}\right)^{M} \mu_{I} \sum_{l=1}^{K} \frac{1}{d_{l}} h\left(f_{l}\left(f_{I}(P)\right)\right)\right]-h(P)+\sum_{I \in W_{M}}\left(\frac{r}{r+1}\right)^{M} \mu_{I} C \\
& \leq\left[\sum_{I \in W_{M}}\left(\frac{r}{r+1}\right)^{M} \mu_{I} \sum_{l=1}^{k} \frac{1}{d_{l}} h\left(f_{l}\left(f_{I}(P)\right)\right)\right]-h(P)+\frac{1}{1-\delta_{S}} C .
\end{aligned}
$$

Let $P$ be a $\Phi$-periodic point and define the height of the images of $P$ by the monoid $\Phi$ to be

$$
h(\Phi(P))=\sup _{R \in \Phi(P)} h(R) .
$$


Since

$$
\sum_{I \in W_{M}}\left(\frac{r}{r+1}\right)^{M} \mu_{I} \sum_{l=1}^{k} \frac{1}{d_{l}}=\left(\frac{r}{r+1}\right)^{M} \sum_{I \in W_{M+1}} \mu_{I}=\left(1+\frac{1}{r}\right) \delta_{S}^{M+1}
$$

and

$$
h(\Phi(P)) \geq h(g(P)) \text { for all } g \in \Phi,
$$

we get

$$
\begin{aligned}
h(P) & \leq\left[\sum_{I \in W_{M}}\left(\frac{r}{r+1}\right)^{M} \mu_{I} \sum_{l=1}^{k} \frac{1}{d_{l}}\right] h(\Phi(P))+\frac{1}{1-\delta_{S}} C \\
& \leq\left(1+\frac{1}{r}\right) \delta_{S}^{M+1} h(\Phi(P))+\frac{1}{1-\delta_{S}} C .
\end{aligned}
$$

By assumption, $\delta_{S}<1$ and $h(\Phi(P))$ is finite, so letting $M \rightarrow \infty$ shows that $h(P)$ is bounded by a constant that depends only on $S$.

\section{References}

[1] S. D. Cutkosky, Resolution of Singularities, Graduate Studies in Mathematics, Vol 63, American Mathematics Society, 2004.

[2] W. Fulton, Intersection Theory, Second edition, Springer-Verlag, Berlin, 1998.

[3] R. Hartshorne, Algebraic Geometry, Springer, 1977.

[4] H. Hironaka, Resolution of singularities of an algebraic variety over a field of characteristic zero. I, Ann. of Math. (2) 79 (1964), 109-203.

[5] S. Kawaguchi, Canonical height functions for affine plane automorphisms, Math. Ann. 335 (2006), no. 2, 285-310.

[6] _ Local and global canonical height functions for affine space regular automorphisms, preprint, arXiv:0909.3573, 2009.

7] S. Lang, Fundamentals of Diophantine Geometry, Berlin, Heidelberg, New York, Springer 1983.

[8] R. Lazarsfeld, Positivity in Algebraic Geometry. I, Ergebnisse der Mathematik und ihrer Grenzgebiete 3. Bd. 48, Springer, New York, 2004.

[9] C. Lee, The upper bound of height and regular affine automorphisms on $\mathbb{A}^{n}$, submitted, arXiv:0909.3107, 2009.

[10] _ The maximal ratio of coefficients of divisors and an upper bound for height for rational maps, submitted, arXiv:1002.3357, 2010.

[11] S. Marcello, Sur la dynamique arithmetique des automorphismes de l'espace affine, Bull. Soc. Math. France 131 (2003), no. 2, 229-257.

[12] D. G. Northcott, Periodic points on an algebraic variety, Ann. of Math. (2) 51 (1950), $167-177$.

[13] I. Shafarevich, Basic Algebraic Geometry, Springer, 1994.

[14] J. H. Silverman, Height bounds and preperiodic points for families of jointly regular affine maps, Pure Appl. Math. Q. 2 (2006), no. 1, part 1, 135-145.

[15] _ The Arithmetic of Dynamical Systems, Springer, 2007.

[16] J. H. Silverman and M. Hindry, Diophantine Geometry, Springer 2000.

[17] A. Weil, Arithmetic on algebraic varieties, Ann. of Math. (2) 53 (1951), 412-444. 
Department of Mathematics

Brown UNIVERSITY

Providence RI 02912, USA

E-mail address: phiel@math.brown.edu 